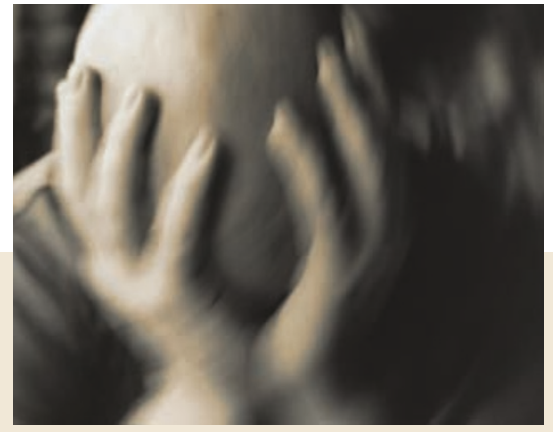

\section{FAAH better anxiolytics?}

The cannabinoid receptor (CB1), the $\mathrm{G}$ protein-coupled receptor activated by the psychotropic components of marijuana, is an attractive target for the development of anxiety- and pain-reducing therapies. However, exogenously applied cannabinoid agonists have been found to give rather variable effects, and a more specific route to regulating $\mathrm{CB} 1$ receptor activity might be to find a way of elevating the levels of the endogenous amidated lipids that activate CB1 receptors. Hence there is interest in targeting the principal degradative enzyme for this group of endogenous $\mathrm{CB} 1$ receptor agonists, the membrane-bound fatty acid amide hydrolase (FAAH), and to this end recently published papers now report the high-resolution crystal structure for FAAH, and a new class of potent and selective FAAH inhibitors.

Transgenic mice lacking FAAH have previously been shown to have increased levels of anandamide, the main amidated lipid candidate for the role of the 'endogenous cannabinoid', and to show enhanced CB1 receptor-dependent analgesia (see further reading). Now, in the November $29^{\text {th }}$ issue of Science, Bracey et al. present the $2.8 \AA$ crystal structure of a slightly truncated version of FAAH, complexed with methoxy arachidonyl fluorophosphonate, an active-site-directed inhibitor. The crystallized FAAH variant, despite having the first 29 amino-terminal amino acids deleted, was reported to retain the wild-type enzyme's association with the cell membrane and ability to degrade fatty acid amides. The crystal structure reveals a dimeric enzyme, closely related to from the amidase signature family for which a structure has been determined and which hydrolyses malonamide in the nitrogen-fixing bacterium Bradyrhizobium japonicum. Interestingly, the close association found in FAAH between the active site and the cell membrane indicates that membrane-permeable lipids, such as anandamide, might not need to be transported through aqueous cellular compartments in order to be degraded, and therefore that the level of endocannabinoid activity might be controlled by the relative expression levels and placement of FAAH and CB1 receptors in vivo.

Knowing the structure of FAAH may help with the future design of inhibitors, but meanwhile a paper from Daniele Piomelli's group, published online in December's Nature Medicine, reports the discovery of the first malonamidase (MAE2), the other enzyme selective inhibitors of FAAH. The two most potent compounds in this class of carbamate inhibitors, URB597 and URB532, were able to elicit anxiolytic-like responses in rats without evoking many of the side effects that normally accompany cannabinoid action, and also produced mild anti-nociception in a model of acute pain. FAAH inhibition was shown to result in increased levels not just of anandamide and other amidated lipids that activate $\mathrm{CB} 1$ receptors, but also of analogues that act independently of $\mathrm{CB} 1$ receptors. So although apparently a new route to the treatment of anxiety by enhancing cannabinoid receptor activation, targeting FAAH also seems likely to affect signalling pathways other than those mediated by $\mathrm{CB} 1$ receptors.

Adam Smith

(i) References and links

ORIGINAL RESEARCH PAPERS Bracey, M.H. et al.

Structural adaptations in a membrane enzyme that terminates endocannabinoid signaling. Science $\mathbf{2 9 8}$ 1793-1796 (2002) | Kathuria, S. et al. Modulation of anxiety through blockade of anandamide hydrolysis. Nature Med. 20022 Dec (doi:10.1038/nm803)

FURTHER READING Cravatt, B.F. et al. Supersensitivity to anandamide and enhanced endogenous cannabinoid signaling in mice lacking fatty acid amide hydrolase. Proc. Natl Acad. Sci. USA 98, 9371-9376 (2001)

\section{DIABETES}

\section{How DPP-IV} takes a bite

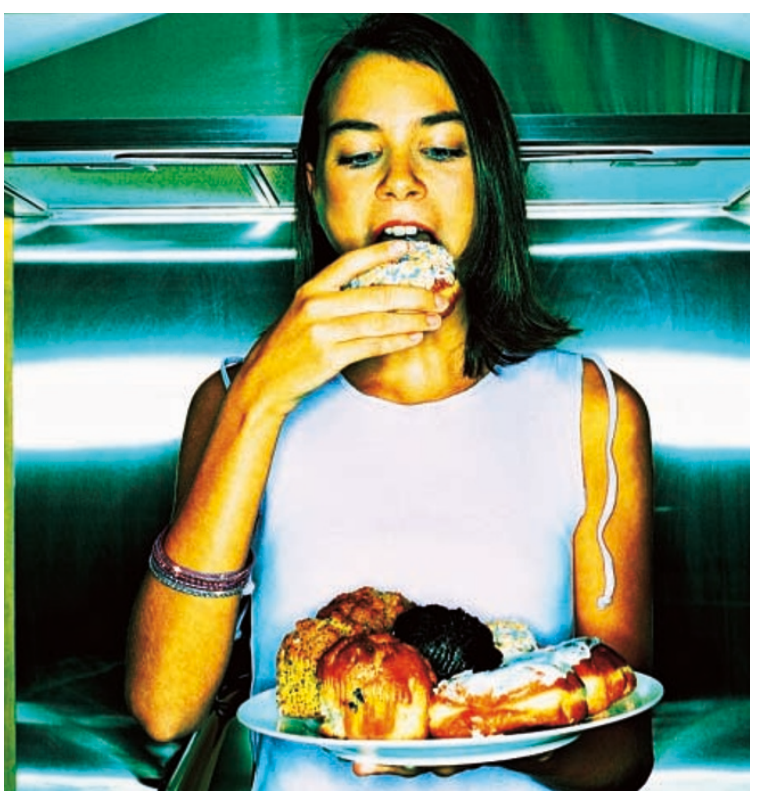

Dipeptidyl peptidase IV (DPP-IV, also known as CD26), a multifunctional transmembrane serine protease, has been attracting considerable interest in recent years as a potential target for type 2 diabetes, owing to its role in regulating blood sugar, and initial trials of DPP-IV inhibitors in humans have been promising. The structure of DPP-IV in complex with an inhibitor, reported in the January issue of Nature Structural Biology, indicates how substrate specificity is achieved, and should be valuable in understanding the structure-activity relationships of known and future inhibitors, and in achieving high specificity for DPP-IV over other members of the same protein family.

DPP-IV modulates the activity of several neuropeptides, chemokines and peptide hormones by specifically cleaving Xaa-Pro or Xaa-Ala from their amino termini. Cleavage of glucagon-like peptide and glucose-dependent insulinotropic peptide - which together are largely responsible for stimulating insulin secretion by $\beta$-cells in response to food - terminates their action. By delaying this normally rapid degradation, DPPIV inhibitors combat the defects in the effects or production of insulin present in type 2 diabetes. And because the ability of DPP-IV inhibitors to promote insulin release is strongly glucose dependent, the risk of hypoglycaemia, a serious side effect of current therapies, is reduced.

Biochemical evidence indicates that DPP-IV functions as a dimer, and indeed, in the crystal structure of the extracellular part of DPP-IV in complex with the inhibitor valine-pyrrolidide determined by Rasmussen et al., DPP-IV is a dimer. Each monomeric subunit has an $\alpha / \beta$ hydrolase domain and an eight-bladed $\beta$-propeller domain, and both domains participate in inhibitor binding in the active site, as well as in dimerization. Intriguingly, it seems that part of the $\beta$-propeller domain involved in dimerization could act as a 'lid' to the active site if the dimer dissociates, which would provide a structural explanation for the biochemical observations that DPP-IV acts as a dimer, and which could represent a novel opportunity for structure-based drug design to block dimer formation.

(D) References and links

ORIGINAL RESEARCH PAPER Rasmussen, H. B. et al. Crystal structure of human dipeptidyl peptidase IV/CD26 in complex with a substrate analogue. Nature Struct. Biol. 2002 Dec 16 with a substrate analog
(doi:10.1038/nsb882)

FURTHER READING Ahrén, B. et al. Inhibition of dipeptidyl peptidase IV improves metabolic control over a 4-week study period in type 2 diabetes. Diabetes Care 25, 869-875 (2002) | Gorrell, M. D., Gysbers, V. \& McCaughan, G. W. CD26: a multifínctional integral membrane and secreted protein of activated lymphocytes. Scand. J. Immunol. 54, 249-264 (2001) 